\title{
ARTICLE
}

Clinical Study

\section{The ProtecT randomised trial cost-effectiveness analysis comparing active monitoring, surgery, or radiotherapy for prostate cancer}

\author{
Sian M. Noble $\mathbb{D}^{1}$, Kirsty Garfield ${ }^{1,2}$, J. Athene Lane ${ }^{1,2}$, Chris Metcalfe ${ }^{1,2}$, Michael Davis ${ }^{1}$, Eleanor I. Walsh ${ }^{1}$, Richard M. Martin ${ }^{1,3}$, \\ Emma L. Turner ${ }^{1}$, Tim J. Peters ${ }^{1}$, Joanna C. Thorn ${ }^{1}$, Malcolm Mason ${ }^{4}$, Prasad Bollina ${ }^{5}$, James W. F. Catto ${ }^{6}$, Alan Doherty ${ }^{7}$, \\ Vincent Gnanapragasam ${ }^{8,9}$, Owen Hughes ${ }^{10}$, Roger Kockelbergh ${ }^{11}$, Howard Kynaston ${ }^{4}$, Alan Paul ${ }^{12}$, Edgar Paez ${ }^{13}$, Derek J. Rosario ${ }^{14}$, \\ Edward Rowe ${ }^{15}$, Jon Oxley ${ }^{16}$, John Staffurth ${ }^{4}$, David E. Neal ${ }^{17}$, Freddie C. Hamdy ${ }^{17}$ and Jenny L. Donovan ${ }^{1}$
}

BACKGROUND: There is limited evidence relating to the cost-effectiveness of treatments for localised prostate cancer. METHODS: The cost-effectiveness of active monitoring, surgery, and radiotherapy was evaluated within the Prostate Testing for Cancer and Treatment (ProtecT) randomised controlled trial from a UK NHS perspective at 10 years' median follow-up. Prostate cancer resource-use collected from hospital records and trial participants was valued using UK reference-costs. QALYs (qualityadjusted-life-years) were calculated from patient-reported EQ-5D-3L measurements. Adjusted mean costs, QALYs, and incremental cost-effectiveness ratios were calculated; cost-effectiveness acceptability curves and sensitivity analyses addressed uncertainty; subgroup analyses considered age and disease-risk.

RESULTS: Adjusted mean QALYs were similar between groups: 6.89 (active monitoring), 7.09 (radiotherapy), and 6.91 (surgery). Active monitoring had lower adjusted mean costs ( $£ 5913)$ than radiotherapy ( $£ 7361)$ and surgery ( $£ 7519)$. Radiotherapy was the most likely (58\% probability) cost-effective option at the UK NICE willingness-to-pay threshold ( $£ 20,000$ per QALY). Subgroup analyses confirmed radiotherapy was cost-effective for older men and intermediate/high-risk disease groups; active monitoring was more likely to be the cost-effective option for younger men and low-risk groups.

CONCLUSIONS: Longer follow-up and modelling are required to determine the most cost-effective treatment for localised prostate cancer over a man's lifetime.

TRIAL REGISTRATION: Current Controlled Trials number, ISRCTN20141297: http://isrctn.org (14/10/2002); ClinicalTrials.gov number, NCT02044172: http://www.clinicaltrials.gov (23/01/2014).

British Journal of Cancer (2020) 123:1063-1070; https://doi.org/10.1038/s41416-020-0978-4

\section{BACKGROUND}

Treatments recommended for cancer localised within the prostate gland include radical surgery, radiotherapy, and active monitoring/surveillance, where radical treatment is avoided or delayed unless/until the cancer shows signs of progression. The ProtecT randomised treatment trial showed there was no evidence of a difference in prostate cancer mortality at a median of 10 years' follow-up between 3D-conformal radiotherapy with neo-adjuvant androgen deprivation therapy (ADT), radical surgery, and active monitoring in men with clinically localised prostate cancer. ${ }^{1}$ Men randomised to surgery and radiotherapy had half the rate of prostate cancer progression and metastasis compared with the active monitoring group, but they experienced greater levels of treatment side effects, including urinary incontinence, erectile dysfunction and bowel symptoms. ${ }^{2}$

In most high- and middle-income countries, healthcare priorities are considered in the context of treatment effectiveness and its cost. Of two trials that compared surgery with watchful waiting, ${ }^{3,4}$ only the Scandinavian Prostate Cancer Group-4 trial (SPCG-4), conducted in the pre-PSA (Prostate Specific Antigen) era, evaluated costs. SPCG-4 found costs $34 \%$ higher for surgery compared with watchful waiting at a median of 12 years' followup. The higher costs for surgery related to the initial treatment costs. $^{3}$ In the absence of more contemporary trial data, models

\footnotetext{
${ }^{1}$ Bristol Medical School, University of Bristol, Bristol, UK; ${ }^{2}$ Bristol Randomised Trials Collaboration, Bristol Trials Centre, University of Bristol, Bristol, UK; ${ }^{3}$ National Institute for Health Research, Bristol Biomedical Research Centre, University of Bristol, Bristol, UK; ${ }^{4}$ The School of Medicine, University of Cardiff, Cardiff, UK; ${ }^{5}$ Department of Urology and Surgery, Western General Hospital, Edinburgh, UK; ${ }^{6}$ The Academic Urology Unit, University of Sheffield, Sheffield, UK; ${ }^{7}$ Department of Urology, Queen Elizabeth Hospital, Birmingham, UK;

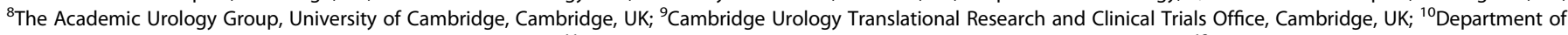
Urology, Cardiff and Vale University Health Board, Cardiff, UK; ${ }^{11}$ Department of Urology, University Hospitals Leicester, Leicester, UK; ${ }^{12}$ Department of Urology, Leeds Teaching Hospitals NHS Trust, Leeds, UK; ${ }^{13}$ Department of Urology, Freeman Hospital, Newcastle-upon-Tyne, UK; ${ }^{14}$ Department of Urology, Sheffield Teaching Hospitals, Sheffield, UK;

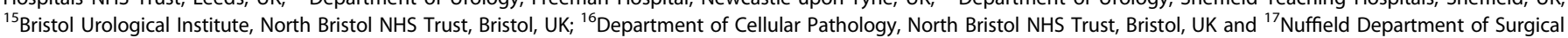
Sciences, University of Oxford, Oxford, UK

Correspondence: Sian M. Noble (s.m.noble@bristol.ac.uk)
}

Received: 7 November 2019 Revised: 8 June 2020 Accepted: 25 June 2020

Published online: 16 July 2020 
1064

have been developed to estimate cost-effectiveness of treatments but have produced conflicting results. ${ }^{5-9}$

This paper presents an individual patient data economic evaluation of the ProtecT trial in terms of costs to the UK NHS and Quality-Adjusted-Life-Years (QALYs) at a median of 10 years' follow-up, the prespecified time point for the primary analysis. ${ }^{1}$

\section{METHODS}

Study design and participants

The ProtecT trial's design and protocol have been published elsewhere. ${ }^{1,10}$ In brief, men in nine UK centres who were detected and diagnosed with clinically localised prostate cancer following a programme of population-based PSA testing who met trial eligibility criteria were randomised to active monitoring ( $n=545)$, surgery $(n=553)$, or radiotherapy with neo-adjuvant ADT $(n=545)$.

Resource-use data collection, coding, and valuation A UK NHS perspective was used for the study; hence, health service resource-use data related to prostate cancer and treatments were recorded from randomisation until November 2015 (a median of 10 years' follow-up). Resource-use relating to initial treatments and subsequent interventions were recorded onto study-specific proformas. Follow-up inpatient stays, outpatient, Emergency Department and, from April 2005, primary care attendances were recorded onto schedules at annual research nurse appointments with participants and from a hospital medical records review. To ensure data completeness, treatment proformas and annual schedules were compared, duplicate data dropped, and combined sources of data used. The trial database containing some clinical information was used for validation purposes. Supplementary Table 1 outlines how the resources were measured, coded and valued (2014-2015 UK $£$ prices) and Supplementary Table 2 outlines how missing resource-use and EQ-5D-3L (QALY) data were handled.

\section{Outcome measurement}

The outcome for the economic analysis is the QALY at a median of 10 years' follow-up. As recommended by NICE, utility values were estimated from the Euroqol EQ-5D-3L questionnaire and associated societal UK utility tariffs. ${ }^{11}$ The EQ-5D-3L was participant-completed prior to diagnosis (baseline), at 6 months, and annually from each man's randomisation date. Men who died were assigned a zero-utility value for the remaining years they could have been trial participants. Utility values were combined to estimate the number of QALYs for each participant using the area-under-the-curve approach. ${ }^{11}$

\section{Analysis}

The analysis compared the three groups as randomised, considering prostate cancer-related NHS costs in relation to QALYs for a median of 10 years. Costs and outcomes for the 10year median analysis were discounted at $3.5 \% .^{12}$ Analyses were conducted in Stata 14.1. ${ }^{13}$ Each item of resource used was summed for each man and the mean resource-use calculated by category (e.g. inpatient stays for infection) and trial group, over the median 10-year period. Each item's cost was calculated as the use (e.g. number of GP visits) multiplied by its unit cost and were summed annually, across time and by resource-use category for each participant.

Annual adjusted mean costs and QALYs were estimated using linear regression. The method of 'seemingly unrelated regressions' (SUR), which accounts for the correlation between costs and QALYs, was used to estimate total adjusted mean costs and QALYs. ${ }^{14}$ Costs and QALYs were adjusted for study centre, age, Gleason score $(<7,7,8-10)$ and PSA at baseline, in keeping with the primary outcome analysis. QALYs were also adjusted for baseline utility. ${ }^{11}$ Across the three treatment groups, the adjusted mean costs and QALYs were compared to assess if any of the treatments were less effective and more expensive than the other treatments. If that was the case, then incremental costeffectiveness ratios (ICERs) would not be estimated in relation to that treatment. ${ }^{15}$ Incremental adjusted mean costs and QALYs, bias-corrected and accelerated confidence intervals (to account for non-normal distributions), and ICERs were estimated using SUR and non-parametric bootstrapping (5000 model iterations). Regression outputs were used to estimate parametrically the incremental net monetary benefit (iNMB) statistic and associated confidence intervals at the UK NICE willingness-to-pay threshold of $£ 20,000$ per QALY. ${ }^{12}$

Cost-effectiveness acceptability curves (CEACs) were generated to explore sampling uncertainty in the cost-effectiveness estimates, ${ }^{16}$ presenting the probability that each trial group is the cost-effective option compared to the other two groups at a range of monetary values. To calculate the CEACs, individual net monetary benefit values were calculated at each willingness to pay per QALY threshold ( $£ 0-£ 100,000$ at $£ 1000$ intervals). At each threshold, 5000 bootstrap model iterations of the adjusted linear regression models of the net monetary benefits were performed. The proportion of times (that is the probability) that each treatment group had the highest net monetary benefit at each threshold was then calculated and plotted to create the CEAC. One-way and scenario sensitivity analyses were used to account for methodological uncertainty or assumptions made during the study and analysis (see Supplementary Information). Exploratory subgroup analyses explored heterogeneity within the study population for age ( $<65$ vs. $65+$ years at randomisation); Grade group ( 1 vs. 2 and higher; and D'Amico risk classification (low vs. intermediate and high). ${ }^{17}$ These were chosen to reflect more relevant contemporary classifications of the prespecified subgroups in the ProtecT primary analysis. ${ }^{1}$

\section{RESULTS}

The adjusted cost and QALY SUR analysis were based on 1101 (67\%) of the 1643 men randomised into the ProtecT trial. More data were available for other analyses (see Table 1, Supplementary Tables 3-4 for sample sizes).

\section{Resource-use}

Resources related to primary treatments varied by randomised groups (Table 1), with the radiotherapy group having more outpatient visits, colonoscopy and sigmoidoscopy procedures, and fewer inpatient stays. There were more primary care resources, biopsies, magnetic resonance imaging (MRI) scans, bone scans, and transurethral resections of the prostate (TURP) in the active monitoring group, and more infection and urinary sphincter-related inpatient stays in the surgery group.

\section{Basecase results}

Over the median 10 years' follow-up, the total adjusted mean costs of the surgery ( $£ 7519$ : $95 \% \mathrm{Cl} £ 7099-£ 7940)$ and radiotherapy ( $£ 7361: 95 \% \mathrm{Cl} £ 6938-£ 7783$ ) groups remained very similar. Both radical groups were more expensive than the active monitoring group (£5913: $95 \% \mathrm{Cl} £ 5494-£ 6332$ ). Year 1 adjusted mean costs were also similar for surgery $(£ 4898)$ and radiotherapy (£4708), but much lower for active monitoring ( $£ 1166$ (Supplementary Table 3; Fig. 1)).

The total adjusted mean QALYs were similar for all groups-just slightly higher for those allocated to radiotherapy (7.093 QALYs: $95 \% \mathrm{Cl}$ 6.914-7.273) compared with active monitoring (6.976 QALYs: $95 \% \mathrm{Cl} 6.798-7.154)$ and surgery $(6.909$ QALYs: $95 \% \mathrm{Cl}$ 6.731-7.087) (Table 2).

The result that the radiotherapy group was more expensive than the active monitoring group but had slightly higher QALYs 
Table 1. Total unadjusted mean resource-use and cost by allocation arm.

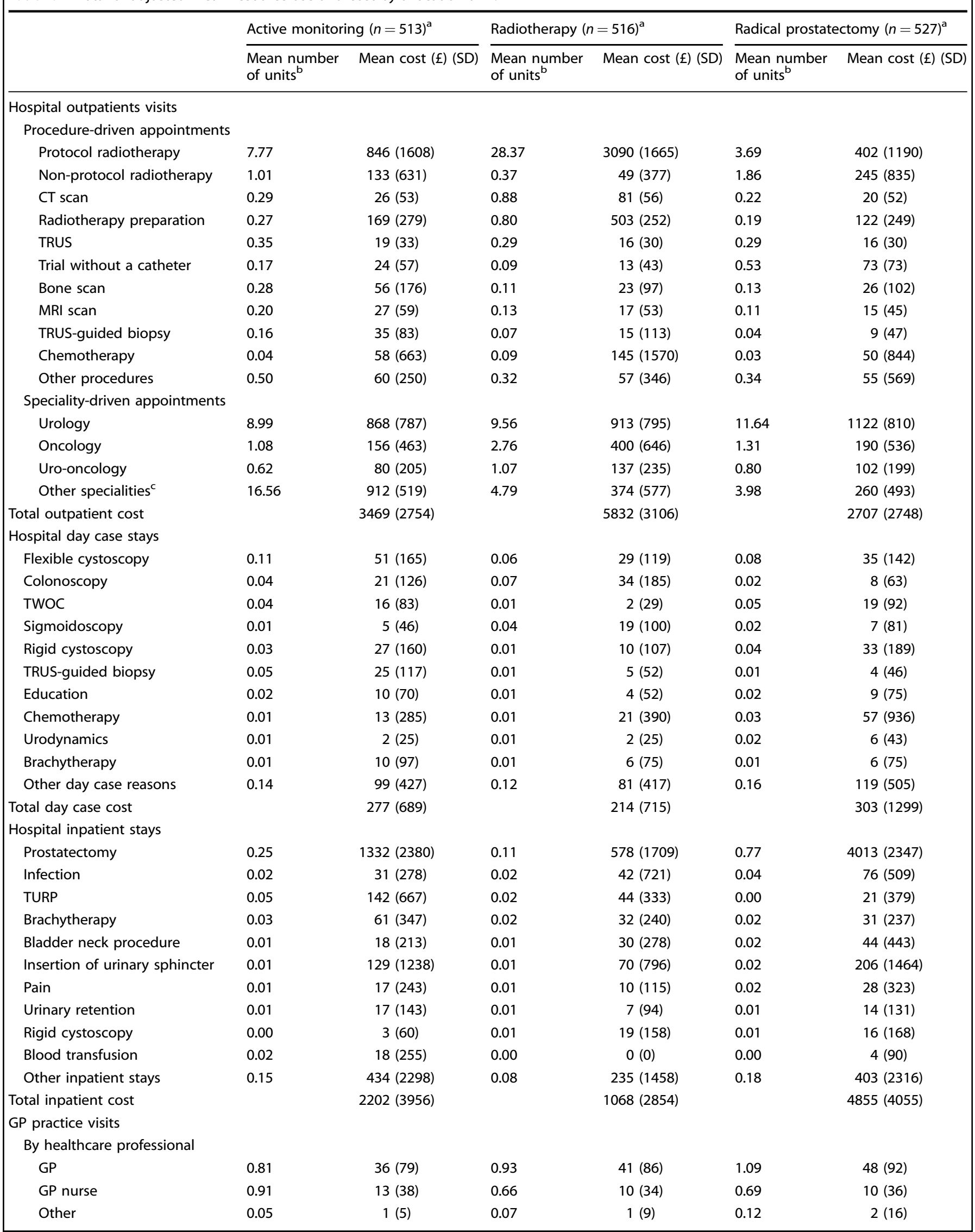


Table 1 continued

\begin{tabular}{|c|c|c|c|c|c|c|}
\hline & \multicolumn{2}{|c|}{ Active monitoring $(n=513)^{a}$} & \multicolumn{2}{|c|}{ Radiotherapy $(n=516)^{\mathrm{a}}$} & \multicolumn{2}{|c|}{ Radical prostatectomy $(n=527)^{\mathrm{a}}$} \\
\hline PSA test & 27.41 & $435(142)$ & 20.91 & $332(140)$ & 20.80 & 330 (138) \\
\hline Hormone delivery & 1.97 & $44(146)$ & 4.24 & $94(87)$ & 0.85 & $19(87)$ \\
\hline Cyproterone acetate days $^{\mathrm{d}}$ & 0.23 & $1(5)$ & 0.37 & $1(7)$ & 0.16 & $0(4)$ \\
\hline Hormone injections & 2.48 & $277(770)$ & 3.96 & $331(515)$ & 1.20 & $154(606)$ \\
\hline Total medication cost & & $277(770)$ & & $332(515)$ & & $154(606)$ \\
\hline Total cost & & $6754(5597)$ & & $7923(4717)$ & & $8428(5636)$ \\
\hline
\end{tabular}

a

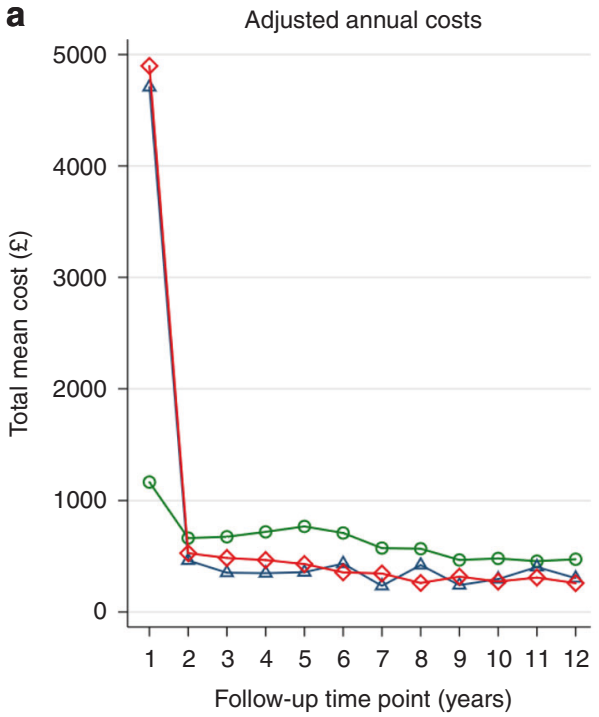

b Adjusted annual QALYs

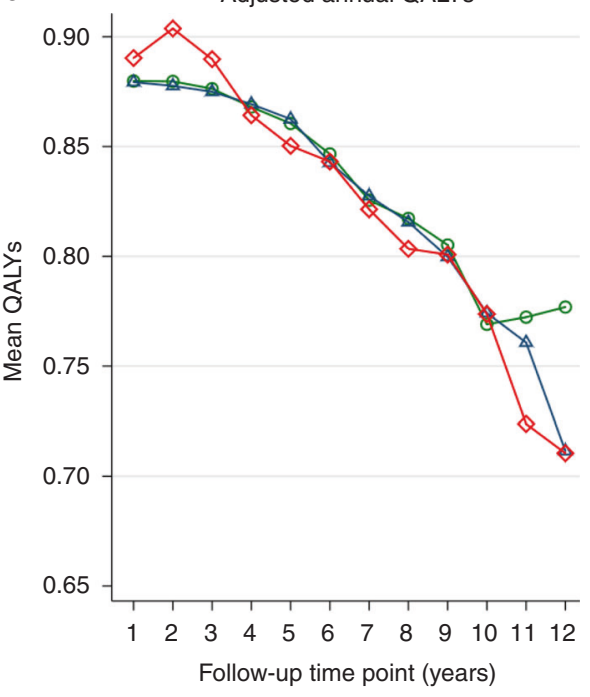

$-\diamond \quad$ Active monitoring
$\curvearrowright \quad$ Radical prostatectomy

$\triangle$ Radiotherapy

Fig. 1 Line charts displaying for each annual follow-up time point, by allocation arm (Active monitoring, Radiotherapy, Radical prostatectomy) the (a) mean adjusted annual costs and (b) mean adjusted annual QALYs (see Supplementary Tables 3 and 4 for sample sizes).

meant that it was appropriate to estimate an ICER. The ICER of $£ 12,310$ per QALY showed that radiotherapy was the cost-effective option at the standard UK NICE willingness-to-pay threshold of $£ 20,000$ per QALY. The CEAC (Fig. 2), reflecting sample uncertainty, shows at this threshold the probability that radiotherapy is the cost-effective option is $58 \%$, with a probability of $32 \%$ for active monitoring and $10 \%$ for surgery.

\section{Sensitivity analyses}

Sensitivity analyses (Supplementary Table 5) confirmed these results for the most part, except when QALY data were not imputed, or when only including participants following the introduction of primary care data collection, or excluding men recruited during the feasibility period; and the scenario using newer techniques (robotic surgery and intensity-modulated radiation therapy (IMRT)). In these four analyses, radiotherapy would be cost-effective at the higher UK-NICE willingness-to-pay threshold of $£ 30,000$ per QALY.

Subgroup analyses

Subgroup analyses (Table 3) showed that QALYs were again similar between groups, but costs varied. For example, for younger men and those with lower risk disease (D'Amico classification), active monitoring was less costly and had higher QALYs than the radical groups and so was most likely to be the cost-effective option. However, for older men and those with higher risk disease, radiotherapy was most likely to be the cost-effective option. At the $£ 20,000$ per QALY threshold, the probability that active monitoring was the cost-effective option was $80 \%$ for younger men, $84 \%$ for D'Amico low-risk disease, and $66 \%$ for Grade group 1; and that 


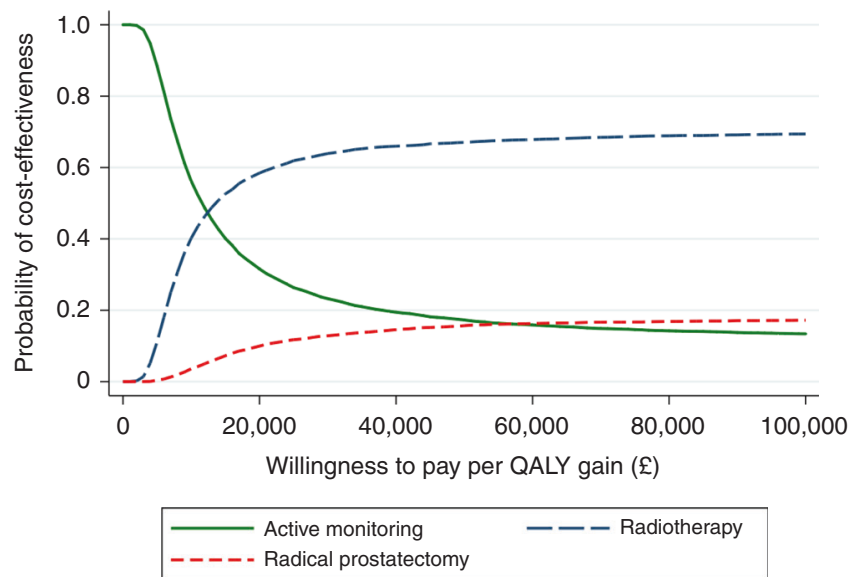

Fig. 2 Cost-effectiveness acceptability curve displaying the probability of each treatment (Active monitoring, Radiotherapy, Radical prostatectomy) being the cost-effective option at different willingness-to-pay thresholds.

radiotherapy was the cost-effective option was $73 \%$ for older men, $69 \%$ for D'Amico intermediate/high-risk disease, and $75 \%$ for Grade group 2 or higher.

\section{DISCUSSION}

In this cost-effectiveness analysis of the ProtecT treatment trial, there were remarkably small differences between the treatment groups at a median of 10 years. Overall, radiotherapy had the greatest probability of being the cost-effective option for localised prostate cancer at a median of 10 years follow-up at the UK NICE willingness-to-pay threshold of $£ 20,000$ per QALY and was most likely to be the cost-effective option at all higher thresholds.

The marginally higher QALYs in the radiotherapy group meant that although it was more costly than the active monitoring group, it was more likely to be the cost-effective option. While the subgroup analyses for both the higher risk, and 65 years and older groups mirrored these results with greater certainty, for younger men and/or those with low-risk disease (defined by the D'Amico classification) active monitoring was more likely to be the costeffective treatment group.

Over the median 10-year period, the mean cost-difference between the radiotherapy and surgery groups was only $£ 159$. The higher surgery group cost was robust to all but the sensitivity analysis that costed robotic surgery and intensity-modulated radiotherapy, and in the subgroup analyses it was lower for younger men and the low-risk groups (range: £127-£244). Throughout all the analyses the surgery group had fewer QALYs than the radiotherapy group; the difference in QALYs would translate into a reduction of 67 days in the best imaginable health over a median of 10 years.

The finding that the active monitoring group had the lowest costs may address clinician concern that active surveillance could be more expensive in the long term because of the need to keep monitoring patients who might ultimately end up having treatment. This study shows that at a median 10 years this was not the case.

The uncertainty reflected in only a $58 \%$ probability that the radiotherapy group was the cost-effective option needs to be acknowledged; the result indicates that it is inconclusive as to which treatment over the median 10 years would be the best value for money. For older men and/or those with higher risk disease, there was more certainty that the radiotherapy group was the cost-effective option; similarly, there was more certainty that 


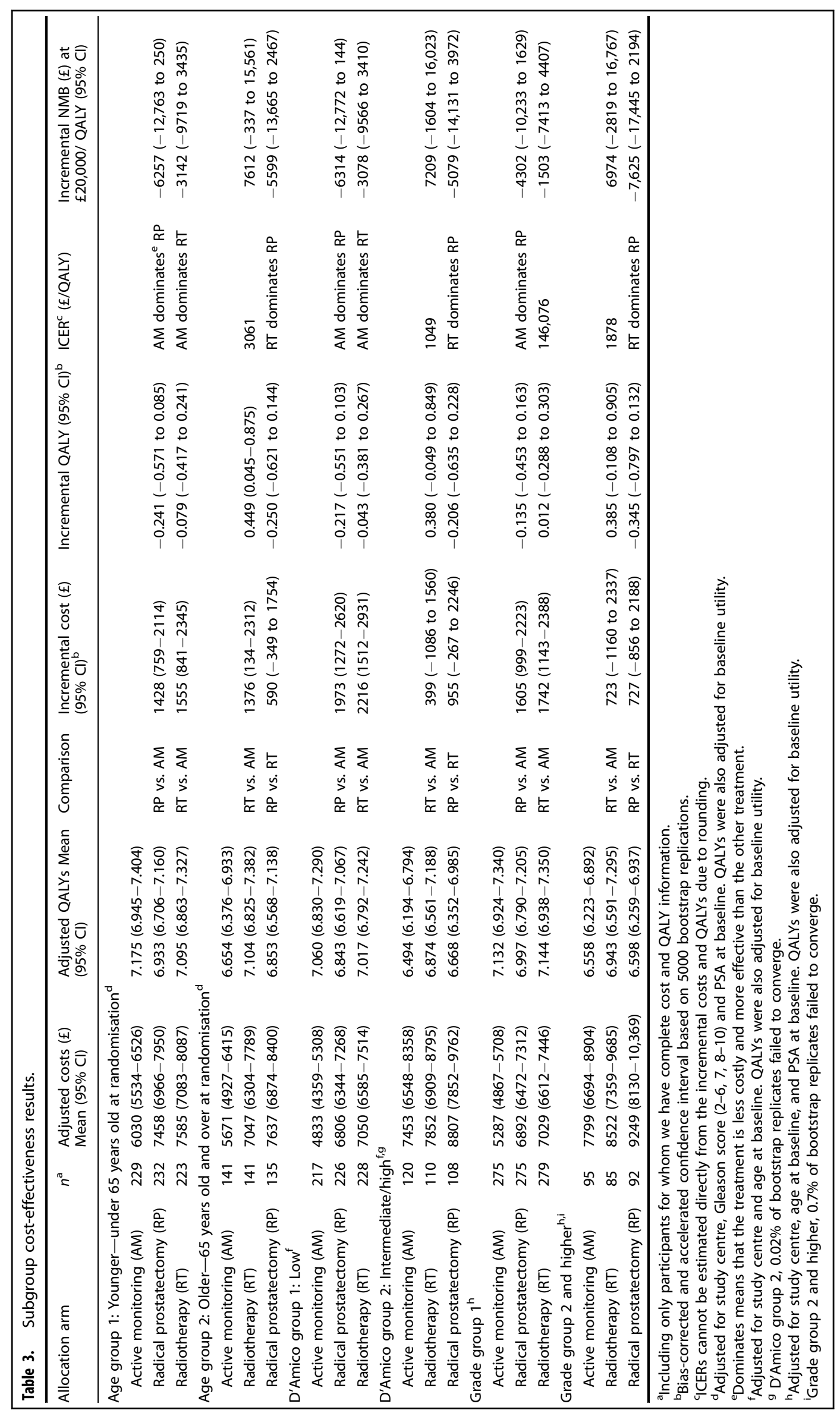


the active monitoring group was the cost-effective option for younger men and/or those with low-risk disease-although these findings, being from subgroup analyses were conducted on smaller numbers and therefore need to be interpreted with some caution.

This study is the first and only economic evaluation within a randomised trial comparing the three contemporary major treatment modalities (ProtecT). The only previous randomised evidence came from SPCG-4, comparing surgery with watchful waiting-not active monitoring, and in the pre-PSA era. This showed higher costs in the surgery arm. $^{3}$ The findings from this study are discordant with some modelling studies. To facilitate comparisons, published costs from the modelling studies were inflated to 2015 prices $^{18}$ and converted to relevant currencies using 2015 purchasing power parities. ${ }^{19}$ Two lifetime modelling studies based on 65-year-old men indicated that active surveillance was the most cost-effective strategy compared with radical prostatectomy (7.6 QALYs: $€ 9585$ ( $£ 9260$ vs. 7.56 QALYs: $€ 16,468$ $(£ 15,911)^{8}$ and 8.85 QALYs: US\$39,894 (£28,796) vs. 7.95 QALYs: US $\left.\$ 38,180(£ 27,559)^{6}\right)$ and IMRT $\left(8.10\right.$ QALYs: US\$48,699 $\left.(£ 35,152)^{6}\right]$. In two models using SPCG-4 data, surgery was shown to be costeffective, although it was more expensive than watchful waiting for 65-year-old men (ICER: 58,045 SEK (£4957) per QALY) ${ }^{20}$ and when an active surveillance programme was considered (ICER: NZ $\$ 33,160(£ 16,070)) .{ }^{9}$ The limited availability of outcome data has been acknowledged to have been a weakness of the models produced. $^{6,8}$

The main strength of this study is that it is based on individual patient data from the ProtecT randomised trial comparing the three contemporary major treatment modalities over a 10-year median duration. The use of medical records in conjunction with a participant visit meant that hospital-based missing data were minimised and likely to be missing completely at random. The presentation of resources used and their costs, and the use of different willingness-to-pay per QALY thresholds, means that country-specific costs and thresholds can be applied to these data.

Limitations of the study include changes that have occurred during the study's duration. New techniques such as robotassisted surgery and IMRT have become more prevalent and are potentially more expensive; in addition, there has been a global shift to shorter treatment courses of radiotherapy. A sensitivity analysis in which surgery was costed as robot-assisted, and radiotherapy as intensity-modulated radiotherapy meant that the radiotherapy group was only cost-effective at the higher $£ 30,000$ per QALY threshold; however, reducing the number of fractions delivered led to the radiotherapy arm becoming more costeffective. Active surveillance programmes have also changed over time. Improved diagnostic techniques (mpMRl) might enable better selection of patients, which could lead to fewer changing to radical treatments or developing metastases, potentially lowering costs and improving effectiveness.

Another important limitation relates to the small number of events in the Protect trial to date because of the long natural history of prostate cancer, meaning that longer follow-up is needed to establish whether there are differences in mortality between the groups-which could change the balance of costeffectiveness. In the late 1990s when the ProtecT trial was being designed, a 10-year time horizon was expected to show differences in clinical events. The exploratory subgroup analyses suggested differences in costs and effectiveness between age and prostate cancer risk groups. It would thus be premature to imply ruling out any of the treatment options at the present time, particularly following the 23-year follow-up of the SPCG-4 trial demonstrating improved survival in men receiving surgery compared with watchful waiting. ${ }^{21}$ Longer-term follow-up is needed in ProtecT to ascertain survival differences.

Other limitations relate to data collection and analysis. Primary care resource-use was not recorded in the follow-up schedules at the beginning of the trial. Therefore, all neo-adjuvant ADT treatment and PSA tests recorded in the trial database were costed. A sensitivity analysis including only participants with all primary care visits recorded indicated that the radiotherapy group was only cost-effective at the higher $£ 30,000$ per QALY threshold. Missing data occurring within the follow-up proformae were imputed following discussions with clinical staff, and a researcher blinded to treatment allocation removed duplicated events between sources, which could have led to small errors. Incomplete EQ-5D-3L data meant QALYs could only be calculated for $69 \%$ of the sample and could have been missing not at random, for example due to ill health. There is a suggestion that participants missing from the surgery group may have been less healthy at baseline than those missing in the other two arms (Supplementary Table 6), potentially leading to an overestimate in the mean QALY reported for the surgery group, but not changing the overall findings.

\section{CONCLUSION}

In the primary economic analysis at a median of 10 years, radiotherapy was the most likely cost-effective option because of slightly lower initial costs and slightly more QALYs, but this result was not conclusive. In subgroup analyses, there was more certainty that radiotherapy was the cost-effective option for older men and/or those with intermediate/high-risk disease. Active monitoring was the least costly option overall, and subgroup analyses suggested it was the most likely cost-effective option for younger men and/or those with low-risk disease. This evaluation provided evidence of the cost-effectiveness of the major primary treatment modalities for clinically localised prostate cancer over a median 10-year period. Further follow-up and subsequent modelling are required to compare other types of treatments, including different radiotherapy modalities, and to assess the impact of later stages of progressing disease to establish which treatment might be most cost-effective over a man's lifetime. In the meantime, all treatments should continue to be offered, with patients, clinicians, and policy-makers using these results in combination with the evidence from ProtecT in relation to the trade-offs between disease progression, metastases, and urinary, sexual and bowel function.

\section{ACKNOWLEDGEMENTS}

We acknowledge the contribution of all ProtecT participants, researchers and clinical staff. The views expressed in this article are those of the authors and do not necessarily reflect the opinions of the National Health Service, the National Institute for Health Research, or the Department of Health. The manuscript has not been deposited on a pre-print server or presented at a scientific meeting in abstract form. The methods and results (excluding the subgroup analyses) within this manuscript will be published in the National Institute for Health Research, Health Technology Assessment Report, "The ProtecT trial-evaluating the effectiveness of treatments for clinically localised prostate cancer".

\section{AUTHOR CONTRIBUTIONS}

S.M.N. had full access to all of the data in the study and affirms that the manuscript is an honest, accurate, and transparent account of the study being reported; that no important aspects of the study have been omitted; and that any discrepancies from the study as originally planned (and, if relevant, registered) have been explained, and attests that all authors meet authorship criteria and that no others meeting the criteria have been omitted. Concept and design: S.M.N., J.L.D., D.E.N., F.C.H., J.A.L., C.M. Acquisition, analysis, or interpretation of data: S.M.N., J.L.D., E.L.T., C.M., M.D., E.I.W., J.A.L., J.C.T., M.M., P.B., J.W.F.C., A.D., V.G., O.H., R.K., H.K., A.P., E.P., D.J.R., E.R., J.O., J.S., D.E.N., F.C.H. Drafting of the manuscript: S.M.N., K.G., J.L.D., J.A.L. Critical revision of the manuscript for important intellectual content: R.M.M., J.L.D., E.L.T., C.M., E.I.W., J.A.L., S.M.N., J.C.T., T.J.P., M.M., P.B., J.W.F.C., A.D., V.G., O.H., R.K., H.K., A.P., E.P., D.J.R., E.R., J.O., J.S., D.E.N., F.C.H. Analysis: K.G., J.C.T., S.M.N. Obtained funding: F.C.H., J.L.D., D.E.N., J.A.L., S.M.N., R.M.M., T.J.P., M.M. Administrative, technical, or material support: E.L.T., E.I.W., J.A.L., J.C.T. Supervision: S.M.N. 


\section{ADDITIONAL INFORMATION}

Ethics approval and consent to participate The UK's East Midlands (formerly Trent) Multicenter Research Ethics Committee (reference number 01/4/025) gave ethical approval. The study was performed in accordance with the Declaration of Helsinki. All participants provided their informed consent before participating in the study.

\section{Consent to publish Not applicable.}

Data availability On request to the ProtecT study, we will provide a patient deidentified set of EQ-5D-3L scores from Baseline (Biopsy) onwards, mortality information and annual costs at the Outpatient, Inpatient, GP and medication level, for prostate cancer-related research as per informed consent for researchers within the EU.

Competing interests S.M.N., K.G., J.A.L., C.M., M.D., E.I.W., T.J.P., F.C.H., D.E.N. and J.L.D. had financial support from NIHR HTA and J.C.T., R.M.M. and E.L.T. from CRUK for the submitted work; M.M. reports personal fees from Janssen Endocyte and Clovis' and scientific advisor to Ellipsis Pharma and to Oncotherics, outside the submitted work. All other authors declare no conflict of interest.

Funding information The ProtecT trial was funded by project grants 96/20/06 and 96/20/99 from the UK National Institute for Health Research, Health Technology Assessment Programme. R.M.M. is supported in part by the National Institute for Health Research Bristol Biomedical Research Centre and by a Cancer Research UK (C18281/A19169) programme grant (the Integrative Cancer Epidemiology Programme). R.M.M., E.L.T., J.C.T. and E.I.W. are supported in part through the CAP trial, which is funded by Cancer Research UK and the UK Department of Health (C11043/ A4286, C18281/A8145, C18281/A11326, C18281/A15064, and C18281/A24432). J.A.L., C.M. and K.G. are supported in part by the Bristol Randomised Trials Collaboration (BRTC), a UKCRC registered clinical trials unit which, as part of the Bristol Trials Centre, is in receipt of National Institute for Health Research CTU support funding.

Supplementary information is available for this paper at https://doi.org/10.1038/ s41416-020-0978-4.

Publisher's note Springer Nature remains neutral with regard to jurisdictional claims in published maps and institutional affiliations.

\section{REFERENCES}

1. Hamdy, F. C., Donovan, J. L., Lane, J. A., Mason, M., Metcalfe, C., Holding, P. et al. 10-year outcomes after monitoring, surgery, or radiotherapy for localized prostate cancer. N. Engl. J. Med. 375, 1415-1424 (2016).

2. Donovan, J. L., Hamdy, F. C., Lane, J. A., Mason, M., Metcalfe, C., Walsh, E. et al. Patient-reported outcomes after monitoring, surgery, or radiotherapy for prostate cancer. N. Engl. J. Med. 375, 1425-1437 (2016).

3. Andersson, S., Andrén, O., Lyth, J., Stark, J. R., Henriksson, M., Adami, H. O. et al. Managing localized prostate cancer by radical prostatectomy or watchful waiting: Cost analysis of a randomized trial (SPCG-4). Scand. J. Urol. Nephrol. 45, 177-183 (2011).

4. Wilt, T. J., Brawer, M. K., Jones, K. M., Barry, M. J., Aronson, W. J., Fox, S. et al. Radical prostatectomy versus observation for localized prostate cancer. N. Engl. J. Med. 367, 203-213 (2012).

5. Eldefrawy, A., Katkoori, D., Abramowitz, M., Soloway, M. S. \& Manoharan, M. Active surveillance vs. treatment for low-risk prostate cancer: a cost comparison. Urologic Oncol.: Semin. Original Investig. 31, 576-580 (2013).
6. Hayes, J. H., Ollendorf, D. A., Pearson, S. D., Barry, M. J., Kantoff, P. W., Lee, P. A. et al. Observation versus initial treatment for men with localized, low-risk prostate cancer: a cost-effectiveness analysis. Ann. Intern. Med. 158, 853-860 (2013).

7. Keegan, K. A., Dall'Era, M. A., Durbin-Johnson, B. \& Evans, C. P. Active surveillance for prostate cancer compared with immediate treatment: an economic analysis. Cancer 118, 3512-2518 (2012).

8. Koerber, F., Waidelich, R., Stollenwerk, B. \& Rogowski, W. The cost-utility of open prostatectomy compared with active surveillance in early localised prostate cancer. BMC Health Serv. Res. 14, 163 (2014).

9. Lao, C., Edlin, R., Rouse, P., Brown, C., Holmes, M. \& Gilling, P. The costeffectiveness of active surveillance compared to watchful waiting and radical prostatectomy for low risk localised prostate cancer. BMC Cancer 17, 529 (2017).

10. Lane, J. A., Donovan, J. L., Davis, M., Walsh, E., Dedman, D., Down, L. et al. Active monitoring, radical prostatectomy, or radiotherapy for localised prostate cancer: study design and diagnostic and baseline results of the ProtecT randomised phase 3 trial. Lancet Oncol. 15, 1109-1118 (2014).

11. Manca, A., Hawkins, N. \& Sculpher, M. J. Estimating mean QALYs in trial-based cost-effectiveness analysis: the importance of controlling for baseline utility. Health Econ. 14, 487-496 (2005).

12. NICE. Guide to the methods of technology appraisal 2013. https://www.nice.org. uk/process/pmg9/chapter/the-reference-case\#framework-for-estimating-clinicaland-cost-effectiveness (2013).

13. StataCorp. Stata Statistical Software: Release 14 (StataCorp LP, College Station, 2015).

14. Greene, W. H. Econometric analysis 5th edn (Prentice Hall, New Jersey, 2002).

15. Gluck, H. A., Doshi, J. A., Sonnad, S. S. \& Polsky, D. Economic Evaluation in Clinical Trials (Oxford University Press, Oxford, 2007).

16. Briggs, A. H. A Bayesian approach to stochastic cost-effectiveness analysis. Health Econ. 8, 257-261 (1999).

17. D'amico, A. V., Whittington, R., Malkowicz, S. B., Schultz, D., Blank, K., Broderick, G. A. et al. Biochemical outcome after radical prostatectomy, external beam radiation therapy, or interstitial radiation therapy for clinically localized prostate cancer. JAMA 280, 969-974 (1998).

18. Inflationtool.com. Inflation calculator. https://www.inflationtool.com/ (2018).

19. OECD. Purchasing power parities (PPP). https://data.oecd.org/conversion/ purchasing-power-parities-ppp.htm\#indicator-chart (2018).

20. Lyth, J., Andersson, S. O., Andren, O., Johansson, J. E., Carlsson, P. \& Shahsavar, N. A decision support model for cost-effectiveness of radical prostatectomy in localized prostate cancer. Scand. J. Urol. Nephrol. 46, 19-25 (2012).

21. Bill-Axelson, A., Holmberg, L., Garmo, H., Taari, K., Busch, C., Nordling, S. et al. Radical prostatectomy or watchful waiting in prostate cancer-29-year follow-up. N. Engl. J. Med. 379, 2319-2329 (2018).

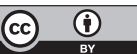

Open Access This article is licensed under a Creative Commons Attribution 4.0 International License, which permits use, sharing, adaptation, distribution and reproduction in any medium or format, as long as you give appropriate credit to the original author(s) and the source, provide a link to the Creative Commons license, and indicate if changes were made. The images or other third party material in this article are included in the article's Creative Commons license, unless indicated otherwise in a credit line to the material. If material is not included in the article's Creative Commons license and your intended use is not permitted by statutory regulation or exceeds the permitted use, you will need to obtain permission directly from the copyright holder. To view a copy of this license, visit http://creativecommons. org/licenses/by/4.0/.

(c) The Author(s) 2020 